EGU2020-12237

https://doi.org/10.5194/egusphere-egu2020-12237

EGU General Assembly 2020

(c) Author(s) 2020. This work is distributed under

the Creative Commons Attribution 4.0 License.

\title{
Improved daily accuracy from a new VIIRS-based, near-real time GFED emissions product
}

Niels Andela, Douglas C. Morton, Guido R. van der Werf, Wilfrid Schroeder, Louis Giglio, Yang Chen, and James T. Randerson

NASA Goddard Space Flight Center, Biospheric Sciences Laboratory, Greenbelt, United States of America

(niels.andela@nasa.gov)

Biomass burning on natural and agricultural lands has profound effects on atmospheric chemistry, climate, and air quality. Over the past decade, a number of global fire emissions inventories have been developed based on near-real time detection of actively burning fires by the MODIS instruments. However, the MODIS instruments provide variable and incomplete global sampling of fire activity, resulting in large uncertainty in the spatiotemporal accuracy of daily emissions inventories. Here, we compared active fire products from MODIS and VIIRS to characterize product-specific shortcomings of each system with the goal to develop a new, more accurate, global emissions inventory. The VIIRS $375 \mathrm{~m}$ product was most sensitive to global fire activity and detected up to $55 \%$ more energy release from fires than the comparable $1 \mathrm{~km}$ MODIS product in the tropics. Differences originated from improved coverage, sensitivity to low energy fires, and a more consistent cross-track spatial resolution. Nevertheless, both MODIS and VIIRS instruments showed reduced sensitivity to low energy fires at larger off-nadir angles, resulting in a cyclical pattern of daily fire detections and an underestimate of low energy fires, the dominant firetype in shoulder seasons and more densely populated regions. Starting in 2018, the constellation of VIIRS instruments aboard NOAA-20 and SNPP provide improved near-nadir coverage, largely eliminating issues originating from incomplete sampling of low-energy fires at the edge of the VIIRS image swath. Based these findings, we developed a new near-real time emissions inventory that is spatially consistent with the GFED4s data record (1997-2016). Spatial allocation of emissions in the new GFED near-real time product differ considerably from existing daily emissions inventories, highlighting how different methodologies redistribute emissions across natural and human dominated landscapes based on daily active fire detections. Using column observations of $\mathrm{NO}_{2}$ and daily fire expansion rates from USGS, we demonstrate that the new, VIIRS-based daily fire emissions product provides more consistent spatial and temporal distribution of fire emissions compared to systems based on MODIS active fire detections. Improved accuracy is critical for air quality forecasts, source attribution, and the development of management strategies to minimize impacts on society. 